\title{
MALDI TIMS IMS of Disialoganglioside Isomers - GD1a and GD1b in Murine Brain Tissue
}

\author{
Katerina V. Djambazova1,2, Martin Dufresne1,3, Lukasz G. Migas', Angela R. S. Kruse ${ }^{2,3}$, Raf Van de \\ Plas$^{4}$, Richard M. Caprioli1 $13,5,6$ and Jeffrey M. Spraggins ${ }^{1-3,7^{*}}$. \\ ${ }^{1}$ Department of Chemistry, Vanderbilt University, 7330 Stevenson Center, Station B 351822, Nashville, TN 37235, USA \\ 2Mass Spectrometry Research Center, Vanderbilt University, $46521^{\text {st }}$ Ave S \#9160, Nashville, TN 37235, USA \\ ${ }^{3}$ Department of Biochemistry, Vanderbilt University, 607 Light Hall, Nashville, TN 37205, USA \\ 4Delft Center for Systems and Control, Delft University of Technology, 2628 CD Delft, The Netherlands \\ 5Department of Pharmacology, Vanderbilt University, 2220 Pierce Avenue, Nashville, TN 37232, USA \\ 6Department of Medicine, Vanderbilt University, $116121^{\text {st }}$ Ave S, Nashville, TN 37232, USA \\ ${ }^{7}$ Department of Cell and Developmental Biology, Vanderbilt University, $46521^{\text {st }}$ Ave S \#3218, Nashville, TN 37232, \\ USA
}

KEYWORDS. Imaging mass spectrometry, Matrix-assisted laser desorption/ionization, Trapped ion mobility spectrometry, Ion mobility mass spectrometry, Gangliosides, Isomers

\begin{abstract}
Gangliosides are classified as acidic glycosphingolipids, containing ceramide moieties and oligosaccharide chains with one or multiple sialic acid residue(s). The presence of multiple sialylation sites gives rise to highly diverse isomeric structures with distinct biological roles. Matrix-assisted laser desorption/ionization imaging mass spectrometry (MALDI IMS) enables the untargeted spatial analysis of gangliosides, among other biomolecules, directly from tissue sections. Integrating trapped ion mobility mass spectrometry (TIMS), a gas-phase separation technology, with MALDI IMS allows for the investigation of isomeric lipid structures in situ. Here we demonstrate the gas-phase separation of disialoganglioside isomers GD1a and GD1b that differ in the position of a sialic acid residue, in a standard mixture of both isomers, a total ganglioside extract, and directly from thin tissue sections. The unique spatial distributions of GD1a/b (d36:1) and GD1a/b (d38:1) were determined from rat hippocampus, as well as in a spinal cord tissue section.
\end{abstract}

Gangliosides are acidic glycosphingolipids, highly abundant in the mammalian nervous system.1,2 They are critical components of neuronal and glial cells, and as such, play an essential role in neuronal development and maturation, as well as cellular signalling. 3,4 These molecules have been implicated in brain injury ${ }^{5}$, as well as in neurological diseases including Guillain-Barre syndrome ${ }^{6,7}$, Alzheimer's ${ }^{8,9}$ and Huntington's ${ }^{10}$ disease, and remain an active area of interest for developing therapeutics.

Gangliosides consist of a hydrophobic ceramide lipid backbone and an oligosaccharide chain of varying length with one (mono-) or many (poly-) sialic acids residues (Figure S1). ${ }^{2}$ The major mammalian brain gangliosides share the same tetrasaccharide core, and have multiple sialylation sites, giving rise to many possible isomeric forms. For example, GM1, GD1a, GD1b, and GT1b share the same tetrasaccharide core (Figure S1) and make up over $80 \%$ of the entire mammalian ganglioside content. ${ }^{11}$ of the highlighted gangliosides, GD1a and GD1b differ in the position of a single sialic acid, where GD1a has a sialic acid on the internal and terminal galactose units, and GD1b has both sialic acids in a chain attached to the internal galactose (Figure S2). In addition to the sialic acid location, the diversity in ceramide/oligosaccharide chain composition also contributed to the large number of ganglioside isomers. This greatly increases the structural complexity and presents a challenge for comprehensive analysis of this class of biomolecules. ${ }^{12}$

Traditionally, ganglioside distributions in murine brain tissues have been characterized using immunohistochemistry (IHC) approaches. These techniques provide carbohydrate epitope information, but have a risk of cross reaction with glycoproteins carrying similar sugar chains. ${ }^{13,14} \mathrm{~A}$ drawback of IHC is that it provides no information on ceramide backbone composition. Since the biological function of gangliosides is dictated by both the carbohydrate chain and the ceramide moieties, analyzing gangliosides in their intact form is crucial for understanding their roles in different physiological processes. ${ }^{11,14,15}$ Mass spectrometry (MS) has been a critical tool for the detection and 
characterization of gangliosides, due to its high specificity and sensitivity. ${ }^{16}$ However, some MS approaches cause extensive precursor ion fragmentation in gangliosides, where the sialic acid links are particularly labile. Soft ionization techniques, including electrospray ionization (ESI) and matrix-assisted laser desorption/ionization (MALDI) ${ }^{17,18}$ have therefore been preferred for ganglioside analysis. ${ }^{11,18}$ MALDI imaging mass spectrometry (IMS) enables highly specific mass analysis while retaining analyte spatial information. ${ }^{19}$ In a typical MALDI IMS experiment, tissues are thinly sectioned, mounted on a conductive glass slide, and coated with a UV-absorbing matrix. An individual mass spectrum is collected at each position (pixel), and molecular images are generated by plotting the ion intensity as a heat map across all the pixels sampled from the tissue. ${ }^{20}$ MALDI IMS has previously been employed to map the localization of gangliosides within biological tissues, including murine and human central nervous system tissues ${ }^{17,21-24}$ However, obtaining comprehensive structural information remains a significant challenge without the use of additional techniques. $^{22-27}$

Liquid chromatography (LC), fragmentation $\left(\mathrm{MS}^{\mathrm{n}}\right)$, and ion mobility separations can be integrated with MALDI IMS to address the structural complexity of gangliosides. ${ }^{28}$ Although highly informative, $\mathrm{MS}^{\mathrm{n}}$ and LC techniques are often highly targeted and/or require lengthy acquisition times. Alternatively, ion mobility enables gas-phase separation of molecules according to their shape, size, and charge on timescales ( $\mu \mathrm{s}-\mathrm{ms}$ ) compatible with typical MALDI IMS throughput. Ion mobility separation techniques, include drift tube ion mobility spectrometry (DTIMS) ${ }^{29,30}$, traveling wave ion mobility spectrometry (TWIMS) ${ }^{31,32}$, structures for lossless ion manipulations (SLIM) ${ }^{33,34}$, field asymmetric ion mobility spectrometry (FAIMS) ${ }^{35-37}$, and trapped ion mobility spectrometry (TIMS). ${ }^{38,39}$ Each of these technologies, with the exception of SLIM, have been coupled to MALDI IMS. ${ }^{40,41}$

Of the mentioned ion mobility approaches, TWIMS MS has been used for in-depth analysis of gangliosides. Clemmer and coworkers have used ESI TWIMS MS for comprehensive untargeted analysis of human brain gangliosides. ${ }^{42-44}$ In the context of structural characterization of ganglioside isomers, Ekroos and coworkers recently demonstrated the separation of isomers GD1a(d36:1) and GD1b(d36:1) using a prototype SLIM-MS instrument. ${ }^{45}$ The isomeric gangliosides were resolved both in a 1:1 mixture, as well as in a wild-type hemi-brain mouse brain extract. ${ }^{45}$ Jackson et. al. have demonstrated the combination of ion mobility and MALDI for the analysis of GD1a and GD1b, where they show higher drift times for the GD1a isomer. ${ }^{26}$ Woods and coworkers have reported the distributions of GD1(d36:1) after a water loss ( $\mathrm{m} / \mathrm{z}$ 1817), to provide some insight into the possible spatial localizations of GD1b. This was possible, as GD1b more readily undergoes a water loss than GD1a. ${ }^{46}$ However, the spatial distributions of $a-$ an $b$ - ganglioside isomers have not been directly demonstrated in a MALDI imaging context thus far.

To our knowledge, the spatial distributions of GD1a/GD1b isomers with ceramide composition information have not been measured directly. Here, we demonstrate the MALDI TIMS IMS analysis of ganglioside isomers GD1a and GD1b from tissue. Briefly, TIMS utilizes an electric field gradient (EFG) applied to an ion tunnel to trap and separate ions against a carrier gas. Following ion trapping, the EFG is gradually reduced to sequentially elute ions with ascending mobilities. ${ }^{38,47}$ Previous MALDI TIMS IMS experiments have demonstrated the utility of the platform for enhanced sensitivity and specificity of lipids imaging experiments, as well as the separation/localization of isobaric and some isomeric phospholipid species in situ. . $^{39,47-52}$

Here we demonstrate the use of MALDI TIMS IMS to distinguish GD1a and GD1b using a 1:1 mixture, a total ganglioside extract, and directly from a thin tissue section. Furthermore, we mapped the unique spatial distributions of GD1a/b(d36:1) and GD1a/b(38:1) in both rat brain and spinal cord samples at $20 \mu \mathrm{m}$ spatial resolution.

\section{METHODS}

Materials: 2',5'-dihydroxyacetophenone (DHA), ammonium sulfate, and anti-GD1 ganglioside antibody were purchased from Sigma-Aldrich (St. Louis, MO, USA). HPLCgrade acetonitrile, methanol, ethanol, and chloroform were purchased from Fisher Scientific (Pittsburgh, PA, USA). Ganglioside extracts were purchased from Avanti Polar Lipids (Alabaster, AL, USA), control rat brain was purchased from BioIVT (Westbury, NY, USA), and control rat spinal cord was purchased from Pel-Freeze Biologicals (Rogers, AZ, USA). goat anti-mouse IgG secondary antibody conjugated to Alexa Fluor Plus 647 was purchased from Invitrogen (Waltham, MA, USA) and DAPI fluoromount-G mounting media was purchased from SouthernBiotech (Birmingham, AL, USA)

Sample Preparation: Control rat brain and spinal cord were cryosectioned to $10 \mu \mathrm{m}$ thickness using a CM3050 S cryostat (Leica Biosystems, Wetzlar, Germany) and thawmounted onto conductive indium tin oxide-coated glass slides (Delta Technologies, Loveland, CO, USA). Matrix deposition protocols were based on Woods and coworkers: 2',5'- DHA with $62.5 \mu \mathrm{M}$ ammonium sulfate in $60 \%$ ethanolwater was sprayed for a final matrix density of 1.23 $\mu \mathrm{g} / \mathrm{mm}^{2}{ }^{24}$ Matrix was applied using a robotic sprayer - M5 Sprayer equipped with a sample heating tray (HTX Technologies, LLC, Chapel Hill, NC, USA). Specific spraying conditions are listed in Table S1. GD1a and GD1b ganglioside extracts (powder) were dissolved in chloroform, aliquoted in vials, dried down with nitrogen, re-dissolved in $80 \%$ methanol for a final extract concentration of $2 \mathrm{mg} / \mathrm{mL}$. The total ganglioside extract had a final concentration of $10 \mathrm{mg} / \mathrm{mL}$. The mixtures were spotted on indium tin oxide-coated glass slides and sprayed with matrix.

MALDI TIMS MS: All experiments were carried out on a prototype MALDI timsTOF Pro mass spectrometer (Bruker Daltonics, Bremen, Germany). ${ }^{50}$ The $1 / \mathrm{K}_{0}$ range, EFG scan time and rate, $m / z$ range, and other specific imaging parameters are listed in Tables S2 and S3. Standards: Data were acquired at $20 \mu \mathrm{m}$ spatial resolution with $\sim 80 \%$ laser power at $10 \mathrm{kHz}, 200$ shots per pixel, and $\sim 300$ pixels per sample. MALDI TIMS IMS specific data are listed in Tables S2 and S3. 
Histology, Microscopy \& Tissue Annotation: Following MALDI IMS experiments, matrix was removed from samples and tissues were stained using a modified cresyl violet stain(Table S4)..$^{53}$ Brightfield microscopy of stained tissues was obtained using a Zeiss AxioScan Z1 slide scanner (Carl Zeiss Microscopy GmbH, Oberkochen, Germany). Annotations of anatomical regions were made on histologically stained tissue sections with the help of The Allen Mouse Brain Atlas ${ }^{54,55}$, The Rat Brain in Stereotaxic Coordinates ${ }^{56}$, and The Atlas of Rat Spinal Cord ${ }^{57}$. Immunofluorescence: Immunofluorescence was performed to verify the localization of GD1a in rat brain and spinal cord tissue sections. Serial tissue sections were fixed with $4 \%$ paraformaldehyde in phosphate buffered saline (PBS) for 10 minutes, followed by blocking in PBS containing 1\% bovine serum albumin and $0.5 \%$ Tween 20 . The sections were hybridized with monoclonal anti-GD1a ganglioside antibody (1:50 dilution) in PBS containing $1 \%$ bovine serum albumin and $0.5 \%$ Tween 20 for 16 hours. Antibody binding was detected using goat anti-mouse IgG secondary antibody conjugated to Alexa Fluor Plus 647 (1:500 dilution) for 1 hour. Tissue sections unexposed to primary antibody were used to determine the level non-specific secondary antibody binding. Sections were mounted with DAPI fluoromount-G mounting media prior to fluorescent imaging. Microscopy was collected using a Zeiss AxioScan Z1, with channels: DAPI, EGFP, and Cy5 (Figures S3 and S4).

Identifications \& Data Processing: Serial tissue sections were analyzed for further structural investigation. To eliminate the possibility of isobaric interference, a tissue sections was analyzed using ultrahigh-spectra resolution FTICR MS (resolving power $\sim 200000$ at $\mathrm{m} / \mathrm{z}$ 1544) (Figure S5). MALDI TIMS MS/MS was used to show diagnostic and preferential a- / b-series fragments from standards (Figure S6), and to confirm ganglioside identification from serial tissue section (Figure S7). MALDI TIMS IMS data were analyzed using DataAnalysis (Bruker Daltonics) and visualized using SCiLS (Bruker Daltonics) and a custom in-house developed software.
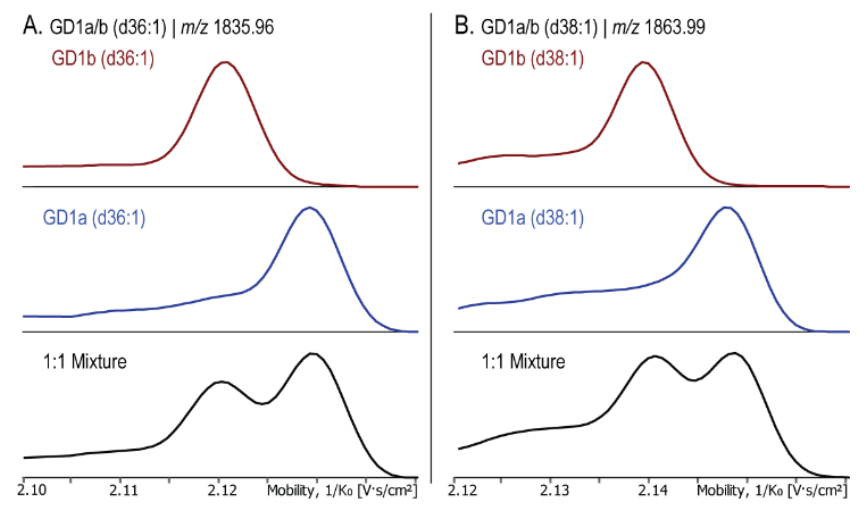

Figure 1. MALDI TIMS extracted ion mobilograms of deprotonated GD1(d36:1): GD1a- (blue) and GD1b- (red), were separated in a 1:1 mixture of both (black) standards (A); and deprotonated GD1(d38:1): GD1a- (blue) and GD1b- (red), were separated in a 1:1 mixture of both (black) standards (B)

\section{RESULTS AND DISCUSSION}

MALDI TIMS analysis of GD1a/GD1b isomer from extracts: Ganglioside extracts were analyzed under two different conditions: high TIMS resolving power (scan rate < $0.01 \mathrm{~V} / \mathrm{ms}$, with a $1100 \mathrm{~ms} \mathrm{t}_{\mathrm{ramp}}$ time), and high throughput (scan rate $-0.03 \mathrm{~V} / \mathrm{ms}$, with a $550 \mathrm{~ms} \mathrm{t}_{\text {ramp }}$ time) (Table S2). Isomeric GD1a and GD1b were first analyzed individually to determine their respective mobilities under MALDI TIMS conditions. The two major GD1 species observed in both GD1a and GD1b standards have ceramide backbones of $\mathrm{d} 36: 1$ and d38:1. Both species were detected as [M-H]; , [M$\left.\mathrm{CO}_{2}-\mathrm{H}\right] ;$; $\left[\mathrm{M}-\mathrm{H}_{2} \mathrm{O}-\mathrm{H}\right]$; , and $[\mathrm{M}+\mathrm{Na}-2 \mathrm{H}]-$ adducts (Figure S8). The deprotonated forms of GD1a/b(d36:1) and GD1a/b(d38:1), detected at $m / z 1835.96$ and $m / z$ 1863.99, respectively, were analyzed to investigate the isomer separation. Under the high TIMS resolving power conditions, the individual $1 / \mathrm{K}_{0}$-values of the deprotonated species GD1b(d36:1) and GD1a(d36:1) were determined to be 1.18 and 1.28, respectively (Figure 1A). From the extracted ion

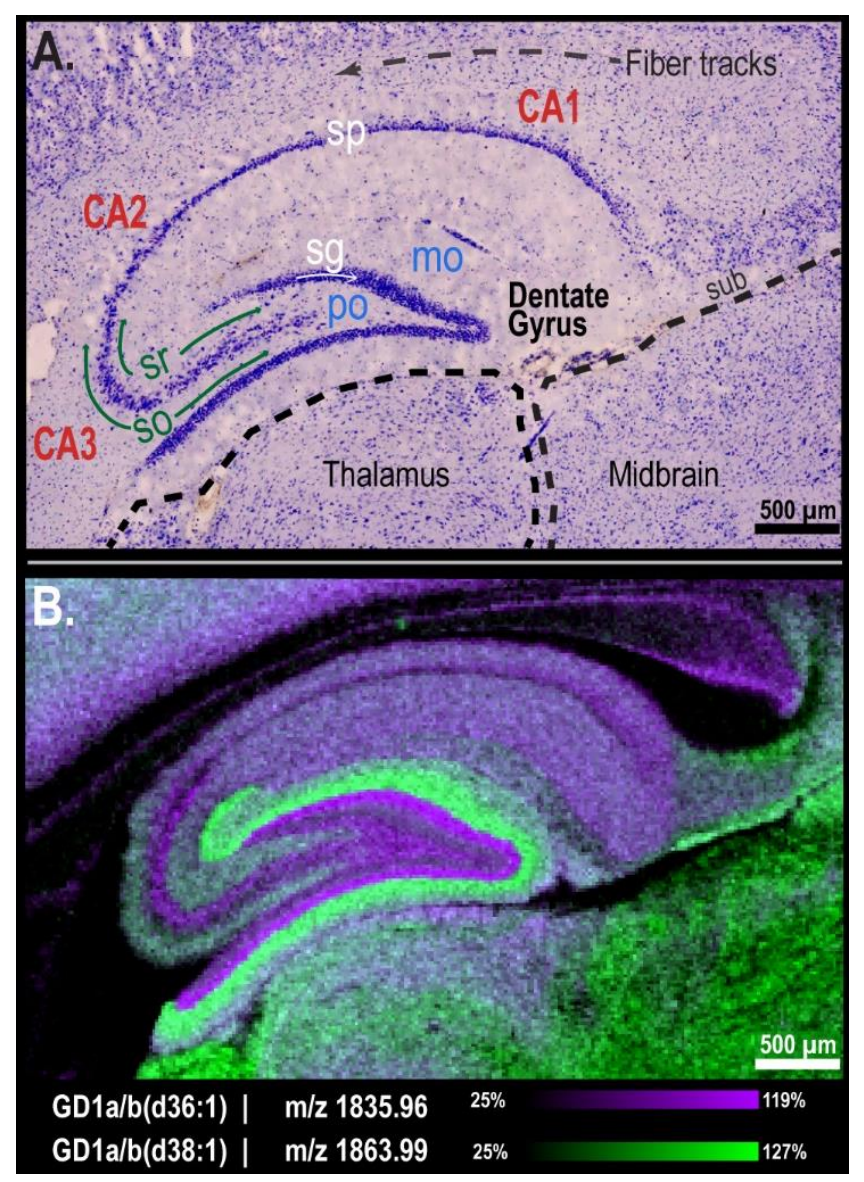

Figure 2. A cresyl violet stain of a sagittal rat brain section highlights features of interest in the hippocampus: Ammon's hors regions - CA1, CA2, and CA3 (in red), and the dentate gyrus, as well as the molecular layer (mo), polymorph layer (po), pyramidal layer (sp), granule cell layer (sg), stratum radiatum (sr), stratum oriens (so), and the subiculum (sub) (A). Overlaid ion images of m/z 1835.96 (purple), and m/z 1863.99 (green), show the different distribution of GD1(d36:1), and GD1(d38:1), respectively, where both distributions are composite of both aand $b$ - series gangliosides (B). 

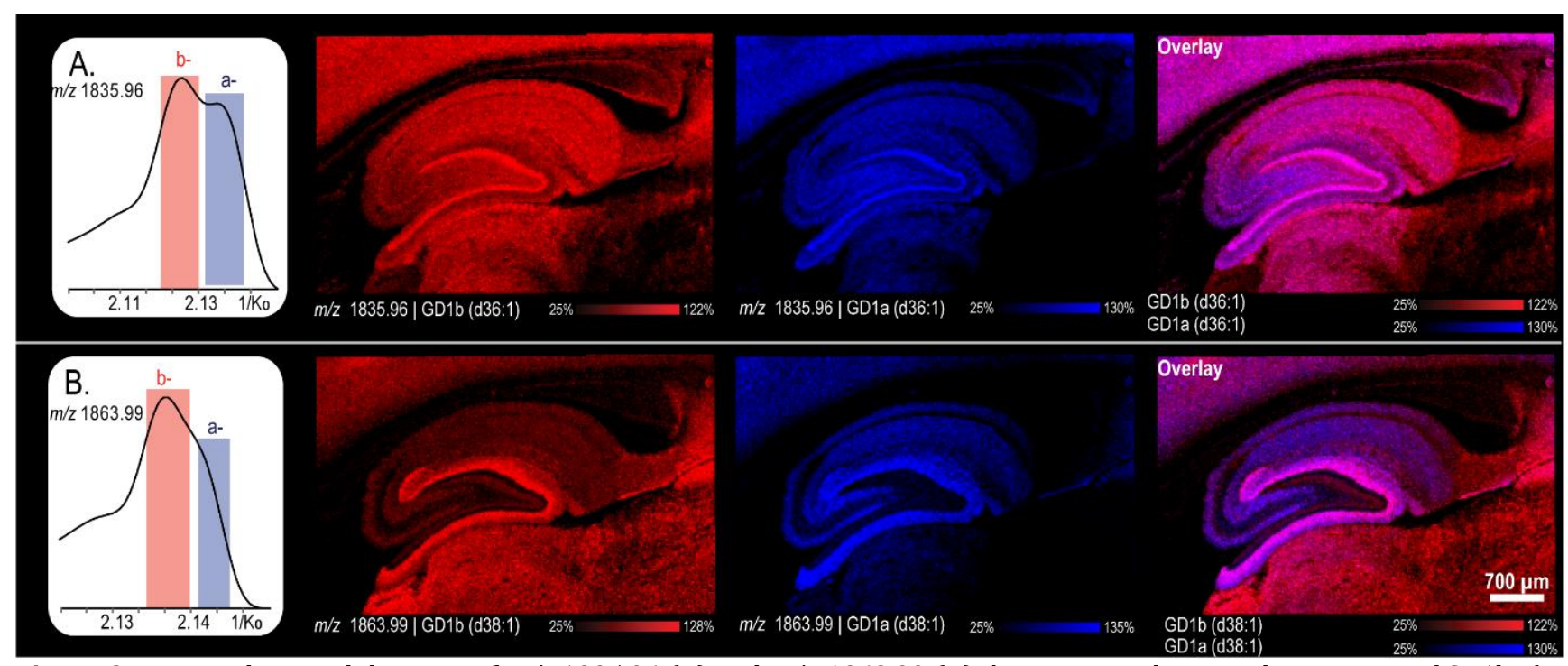

Overlay

Figure 3. Extracted ion mobilograms of $\mathrm{m} / \mathrm{z} 1835.96$ (A) and $\mathrm{m} / \mathrm{z} 1863.99$ (B) demonstrate the partial separation of GD1b- (red) and GD1a (d36:1) (blue) in rat hippocampus tissue section. Ion images of GD1b (red), GD1a (blue) isomers, and an overlay of both ions can be seen for both GD1(d36:1) (A) and GD1(d38:1) (B).

mobilogram of the mixture, a partial separation of the two isomers was observed. Under the same TIMS conditions, the extracted ion mobilograms of GD1b(d38:1) and GD1a(d38:1) determined the individual 1/Ko values to be 1.14 and 1.15, respectively (Figure 1B), where the two isomers were partially resolved in a 1:1 mixture. Additionally, we analyzed a total ganglioside extract demonstrating the partial separation of GD1a/b for both (d36:1) and (d38:1) species (Figure S9). As this data were acquired with the goal of maximizing the TIMS resolving power, the experiment was repeated to show a separation that was more amenable to a MALDI IMS throughput (550 ms $\mathrm{t}_{\text {ramp }}$ time, $600 \mathrm{~ms} / \mathrm{pixel}$ ) (Figure S10). Our findings indicate that GD1b and GD1a isomers can be partially resolved in a 1:1 mixture, and in a total ganglioside extract, where the b-series isomers have more compact structures leading to a lower $1 / \mathrm{K}_{0}$ values. These findings are consistent with previous MALDI-ion mobility experiments by Jackson et. al. ${ }^{26}$, as well as SLIM MS experiments by Ekroos and coworkers. ${ }^{45}$

MALDI TIMS IMS separation and localization of GD1a/GD1b in rat brain and spinal cord tissue.

RAT BRAIN: GD1a and GD1b species are the most abundant ganglioside species in the mammalian brain. ${ }^{46}$ Both aand $b$-series disialogangliosides have distinct localizations within the brain, previously characterized using immunohistochemistry (IHC) approaches. MALDI TIMS IMS data was collected from a sagittal rat brain section (left hemisphere, lateral value $\sim 0.8-0.9 \mathrm{~mm}$ ), at $20 \mu \mathrm{m}$ spatial resolution. The average mass spectrum shows that $\sim \mathrm{m} / z 1800$ 1900 was dominated by the GD1(d36:1) and GD1(d38:1) species, detected as $[\mathrm{M}-\mathrm{H}],\left[\mathrm{M}-\mathrm{CO}_{2}-\mathrm{H}\right],\left[\mathrm{M}-\mathrm{H}_{2} \mathrm{O}-\mathrm{H}\right]$, and $[\mathrm{M}+\mathrm{Na}-2 \mathrm{H}]^{-}$adducts (Figure S2). A cresyl violet stain was used to highlight the regions of interest in the hippocampus that were analyzed by MALDI IMS (Figure 2A). Ion images of $m / z 1835.96$ (purple) and $m / z 1863.99$ (green) show the different distributions of GD1(d36:1) and GD1(d38:1), respectively, in the hippocampus (Figure 2B). Comparing the ion images with the annotated stain, we observed that GD1(d36:1) was more pronounced in the granular layer of the dentate gyrus, the pyramidal layer of the CA3 and CA2 regions, and along the fiber tracks. GD1(d38:1), on the other hand, was more pronounced in the molecular layer of the dentate gyrus, in the midbrain, and along the edges of the subiculum. The overlaid ion images represent a composite image of $a-$ and $b$-series isomers for both 36:1 and 38:1. Further investigation using the TIMS mobility dimension reveled two partially resolved peaks for $m / z$ 1835.96, corresponding to deprotonated GD1a(d36:1) and GD1b(d36:1). Individual standards, GD1a and GD1b, were also acquired using the same conditions to aid in identification (Figure S12). Ion images of each of the ion mobility peaks (Figure 3A) show the unique spatial distributions of both a-(blue) and $b$-(red) series isomers. GD1a and GD1b were expressed in all layers of the hippocampus but differed in their relative intensities in specific subregions. In the ion images we observed that the a-isomer was more pronounces in the granular layer of the dentate gyrus, while the b-isomer was more abundant in the subiculum, as well as the midbrain, and thalamus, where the signal from the a-series was noticeably absent. Similarly, the extracted ion mobilogram of $\mathrm{m} / \mathrm{z}$ 1863.99, highlighted two partially resolved ions, corresponding to a- and b-series GD1(d38:1) (Figure 3B). Although, the two ions were not as well resolved in the gas phase, their localizations within the rat brain tissues were very different. The a-isomer was observed in the granular layer of the dentate gyrus and was more pronounced in the striatum radiata and striatum oriens of the CA3 region. GD1b was also more pronounced in the granular layer of the CA1 and CA2 regions, where GD1a was below the limit of detection. GD1a was, however, present in the striatum radiata and the striatum oriens of CA1 and CA2. Similar to GD1a/b(d36:1), the most significant distinction between the two isomers was the detection of the a-series in the 

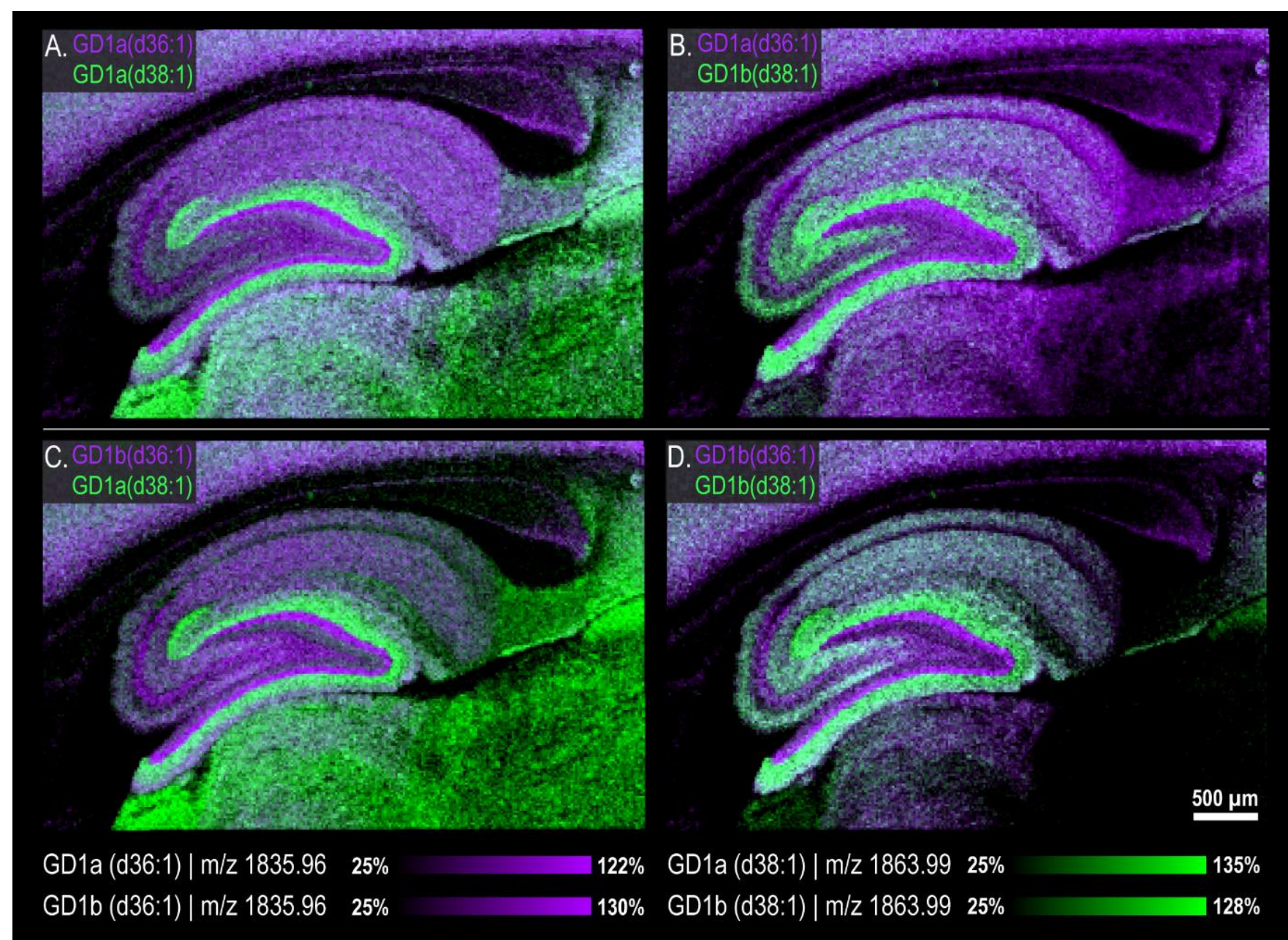

GD1a (d36:1) | m/z $1835.96 \quad 25 \%$ GD1b (d36:1) | m/z $1835.96 \quad 25 \%$
$122 \%$

$130 \%$
GD1b (d38:1) | m/z $1863.9925 \%$

Figure 4. Overlay ion images of a and b-series isomers for GD1(d36:1) and GD1(d38:1): GD1a(d36:1) and GD1a(d38:1) (A), GD1a(d36:1) and GD1b (d38:1) (B), GD1b(d36:1) and GD1a(d38:1) (C), and GD1b(d36:1) and GD1b(d38:1) (D).

thalamus, but not the midbrain or the subiculum. These findings are consistent with previous IHC experiments of mouse and rat brains, showing GD1a and GD1b localizations $^{14,58}$, and with previous IMS data, highlighting the differences between GD1(d36:1) and of GD1(d38:1). ${ }^{24,25}$ Woods and coworkers had previously reported mapping of $\mathrm{GD} 1 \mathrm{~b}(\mathrm{~d} 36: 1)$ by imaging the water-loss at $\mathrm{m} / z$ 1817.95, which is more preferential for the $b$-series gangliosides. ${ }^{46}$ Here we highlight the subtle differences of GD1 subspecies that differ in both ceramide composition and sialic acid localizations. This work shows that with the combination of MALDI IMS and ion mobility separations the spatial distributions of GD1a(d36:1), GD1b (d36:1), GD1a(d38:1), GD1b(d38:1) and any combination of thereof can be visualized directly from thin tissue sections (Figure 4A-D). In addition to the two overlaid ion images in Figure 2, there are four additional ion overlay combinations possible, highlighted in Figure 4. As ganglioside role and function is influenced by the ceramide moieties and the carbohydrate chains, this level of structural specificity in an imaging context is important to further understand their role in biology and disease.
SPINAL CORD: To further highlight the unique distributions of GD1a and GD1b isomers in murine nervous tissue, rat spinal cord tissue was analyzed. A transverse section of the sympathetic intermediolateral horn (roughly T6 - T11 region) was selected, as the a- and b-series GD1 localizations have been previously highlighted in literature by IHC. ${ }^{14}$ A cresyl violet microscopy image was annotated to show the regions of interest (Figure S13). Considering the relatively small size of the tissue section, MALDI TIMS parameters were optimized to maximize the separations, and demonstrate the highest resolution separation of GD1a/b isomers in situ.

The extracted ion mobilograms of the GD1(d36:1) and GD1(d38:1) revealed two isomeric species (Figure 5A and 5B, respectively). From the ion images of $m / z 1835.96$ (Figure 5A) we can see that the b-series was found throughout the gray matter with higher intensity in the rexed laminae 1 and 2 of the dorsal horn. The a-series isomer, on the other hand, was only present in the rexed laminae. The ion images of $m / z 1863.99$ (Figure 5B) revealed that GD1b was detected throughout the gray matter. The a-series isomer was more pronounced in the rexed laminae, but could also be detected in the gray matter, specifically around the central 

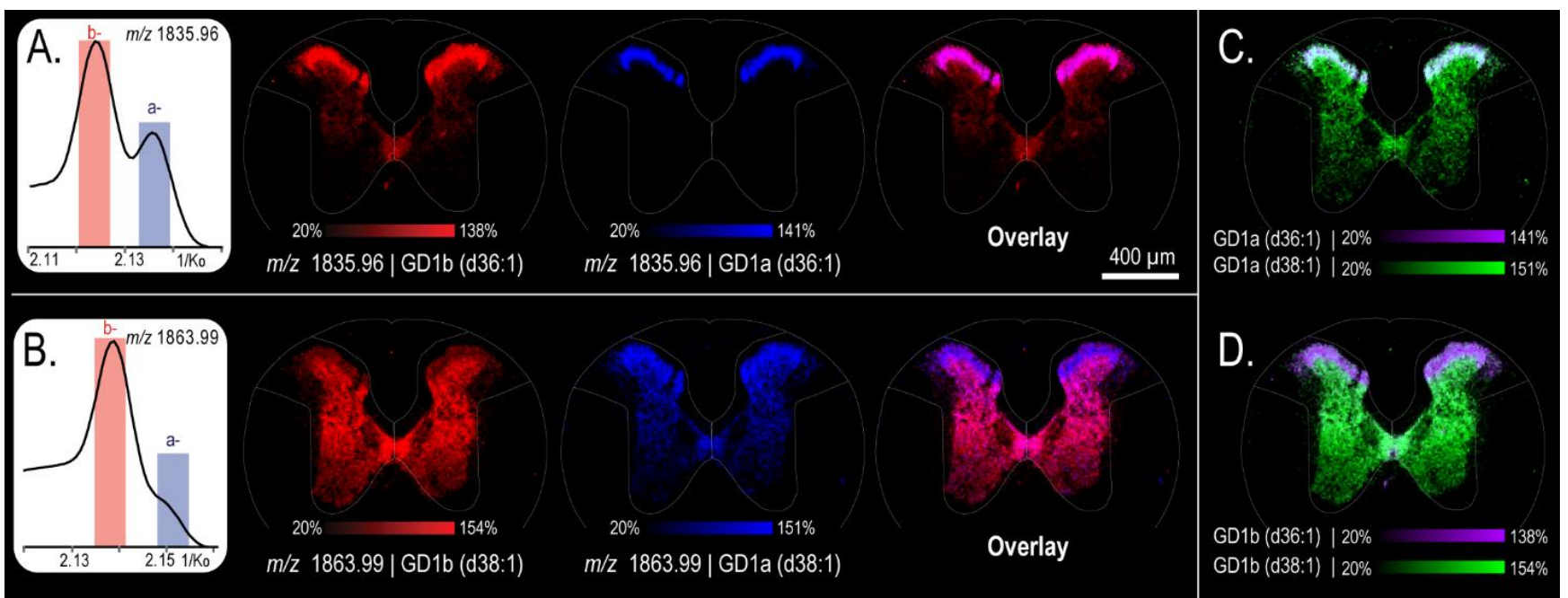

Figure 5. Extracted ion mobilograms of $m / z 1835.96$ (A) and $\mathrm{m} / \mathrm{z} 1863.99$ (B) demonstrate the partial separation of GD1b- (red) and GD1a (d36:1) (blue) in rat spinal cord section. Ion images of GD1b (red), GD1a (blue) isomers, and an overlay of both ion s can be seen for both GD1(d36:1) (A) and GD1(d38:1) (B). Overlay ion images of GD1a (d36:1) and (d38:1), as well as GD1b (d36:1) and (d38:1) are highlighted in (C) and (D), respectively.

canal (Figure 5B). An overlay of GD1b(d36:1) and GD1b(d38:1) (Figure 5C) revealed that the signal in the rexed laminae was mostly due to the $\mathrm{d} 36: 1$, where the $\mathrm{d} 38: 1$ was more pronounced in the gray matter. The overlay of GD1a(d36:1) and GD1a(d38:1) (Figure 5D) shows that GD1a(d36:1) was more intense in the lamiae than the d38:1, which was detected throughout the gray matter.

Our findings are consistent with previous IHC studies that show GD1a and GD1b, and with MALDI IMS studies that show the spatial distribution of GD1(d36:1) vs GD1(d38:1) localization within murine spinal cord tissue. ${ }^{14}$ Neither of the latter two approaches can resolve the nuanced differences in ceramide composition and carbohydrate chain simultaneously. For example, the composite ion images of GD1a(d36:1) and GD1a(d38:1) could not be resolved with traditional IHC approaches. Similarly, mapping the localizations of $\mathrm{GD} 1 \mathrm{a}(\mathrm{d} 36: 1)$ and $\mathrm{GD} 1 \mathrm{~b}(\mathrm{~d} 38: 1)$, as well as GD1b(d36:1) and GD1a(d38:1), could not be achieved with either MALDI IMS, or IHC alone. These examples highlight the utility of MALDI TIMS IMS for the gas-phase separation of GD1a- and b-series isomers in situ.

\section{CONCLUSIONS}

This work demonstrates the capabilities of MALDI TIMS IMS in elucidating the spatial distribution of both GD1a/b(36:1) and GD1a/b(38:1) species in tissue samples which cannot be achieved through standard IHC experiments, nor typical MALDI IMS workflows. We demonstrated the unique spatial distribution of these gangliosides directly from rat brain and spinal cord tissues, using this MALDI TIMS IMS. This level of structural and spatial characterization directly from biological tissue sections may offer new insight in the roles gangliosides play in different physiological processes.

\section{ASSOCIATED CONTENT}

\section{Supporting Information}

Major brain gangliosides' structure (Figure S1); GD1a and GD1b molecular structure (Figure S2); Matrix deposition parameters (Table S1); MALDI TIMS experimental parameters (Table S2); MALDI IMS parameters (Table S3); Cresyl Violet staining procedure (Table S4); MALDI FT-ICR data (Figure S3); Fragmentation spectra of GD1a and GD1b extracts (Figure S4); On-tissue fragmentation spectra of GD1(d36:1) and GD1(d38:1) (Figure S5); Average mass spectrum of GD1a and GD1b extracts (Figure S6); Extracted ion mobilograms from total ganglioside extract (Figure S7); Extracted ion mobilograms for ganglioside isomers with high throughput method (Figure S8)Average mass spectrum of rat hippocampus (Figure S9); Aligned ion mobility of GD1a/b standards and rat brain data (Figure S10); Rat Spinal Cord Annotations (Figure S11);

\section{AUTHOR INFORMATION}

\section{Corresponding Author}

* Jeffrey M. Spraggins, Jeff.Spraggins@vanderbilt.edu Jeffrey M. Spraggins: 0000-0001-9198-5498

Katerina V. Djambazova:0000-0002-2680-9014

Martin Dufresne: 0000-0002-1731-3666

Lukasz G. Migas:0000-0002-1884-6405

Angela R. S. Kruse : 0000-0001-8776-2769

Raf Van de Plas: 0000-0002-2232-7130

Richard M. Caprioli: 0000-0001-5859-3310

\section{Author Contributions}

All authors have given approval to the final version of the manuscript.

\section{ACKNOWLEDGMENT}

Support was provided by The National Science Foundation Major Research Instrument Program (CBET - 1828299 awarded 
to J.M.S. and R.M.C.) The 15T FTICR MS in the Mass Spectrometry Research Center at Vanderbilt University was acquired through the NIH Shared Instrumentation Grant Program (1S100D012359).

\section{REFERENCES}

(1) Svennerholm, L. The Gangliosides. J. Lipid Res. 1964, 5 (2), 145-155. https://doi.org/10.1016/s0022-2275(20)402317.

(2) Yu, R. K.; Tsai, Y. T.; Ariga, T.; Yanagisawa, M. Structures, Biosynthesis, and Functions of Gangliosides-an Overview. J. Oleo Sci. NIH Public Access 2011, pp 537-544. https://doi.org/10.5650/jos.60.537.

(3) Sonnino, S.; Mauri, L.; Chigorno, V.; Prinetti, A. Gangliosides as Components of Lipid Membrane Domains. Glycobiology 2007, 17 (1), 1R-13R. https://doi.org/10.1093/GLYCOB/CWL052.

(4) Lunghi, G.; Fazzari, M.; Biase, E. Di; Mauri, L.; Chiricozzi, E.; Sonnino, S. The Structure of Gangliosides Hides a Code for Determining Neuronal Functions. FEBS Open Biol. 2021. https://doi.org/10.1002/2211-5463.13197.

(5) Benady, A.; Freidin, D.; Pick, C. G.; Rubovitch, V. GM1 Ganglioside Prevents Axonal Regeneration Inhibition and Cognitive Deficits in a Mouse Model of Traumatic Brain Injury. Sci. Rep. 2018, 8 (1), 1-10. https://doi.org/10.1038/s41598018-31623-y.

(6) Kaida, K.; Ariga, T.; Yu, R. K. Antiganglioside Antibodies and Their Pathophysiological Effects on Guillain-Barré Syndrome and Related Disorders-A Review. Glycobiology 2009, 19 (7), 676-692. https://doi.org/10.1093/GLYCOB/CWP027.

(7) Yuki, N.; Suzuki, K. Review Guillain-Barré Syndrome and AntiGanglioside Antibodies: A Clinician-Scientist's Journey. Proc. Jpn. Acad., Ser B Phys . Biol. Sci. 2012, 88 (7), 299-326. https://doi.org/10.2183/pjab.88.299.

(8) Ariga, T.; McDonald, M. P.; Yu, R. K. Role of Ganglioside Metabolism in the Pathogenesis of Alzheimer's Disease - A Review. J. Lipid Res. 2008, 49 (6), 1157-1175. https://doi.org/10.1194/jlr.R800007-JLR200.

(9) Ariga, T.; McDonald, M. P.; Yu, R. K. Thematic Review Series: Sphingolipids. Role of Ganglioside Metabolism in the Pathogenesis of Alzheimer's Disease-a Review. J. Lipid Res. 2008, 49 (6), 1157-1175. https://doi.org/10.1194/JLR.R800007-JLR200.

(10) M, A.; D, G.; J, F.; LC, M.; SW, L.; P, K.; A, D. P.; A, H.; BJ, K.; KG, T.; GB, B.; K, F.; S, S. Disease-Modifying Effects of Ganglioside GM1 in Huntington's Disease Models. EMBO Mol. Med. 2017, 9 (11), 1537-1557. https://doi.org/10.15252/EMMM.201707763.

(11) Metelmann, W.; Vukelić, Ž.; Peter-Katalinić, J. NanoElectrospray Ionization Time-of-Flight Mass Spectrometry of Gangliosides from Human Brain Tissue. J. Mass Spectrom. 2001, 36 (1), 21-29. https://doi.org/10.1002/JMS.100.

(12) Hájek, R.; Jirásko, R.; Lísa, M.; Cífková, E.; Holčapek, M. Hydrophilic Interaction Liquid Chromatography-Mass Spectrometry Characterization of Gangliosides in Biological Samples. Anal. Chem. 2017, 89 (22), 12425-12432. https://doi.org/10.1021/acs.analchem.7b03523.

(13) Kotani, M.; Kawashima, I.; Ozawa, H.; Terashima, T.; Tai, T. Differential Distribution of Major Gangliosides in Rat Central Nervous System Detected by Specific Monoclonal Antibodies. $\begin{array}{llll}\text { Glycobiology } & 1993, & 3 & \text { (2), 137-146. }\end{array}$ https://doi.org/10.1093/GLYCOB/3.2.137.

(14) K, V.; B, V.; IV, D.; RL, S.; M, H. Differential Distribution of Major Brain Gangliosides in the Adult Mouse Central Nervous System. PLOS ONEne 2013, 8 (9). https://doi.org/10.1371/JOURNAL.PONE.0075720.

(15) Li, Z.; Zhang, Q. Ganglioside Isomer Analysis Using Ion Polarity Switching Liquid Chromatography-Tandem Mass Spectrometry. Anal. Bioanal. Chem. 2021, 413 (12), 32693279. https://doi.org/10.1007/S00216-021-03262-2.

(16) O'connor, P. B.; Mirgorodskaya, E.; Costello, C. E. High
Pressure Matrix-Assisted Laser Desorption/Ionization Fourier Transform Mass Spectrometry for Minimization of Ganglioside Fragmentation. J. Am. Soc. Mass Spectrom. 2002, $13(4), 402-407$.

(17) Zhang, Y.; Wang, J.; Liu, J.; Han, J.; Xiong, S.; Yong, W.; Zhao, Z. Combination of ESI and MALDI Mass Spectrometry for Qualitative, Semi-Quantitative and in Situ Analysis of Gangliosides in Brain. Sci. Rep. 2016, 6 (1), 1-11. https://doi.org/10.1038/srep25289.

(18) Ito, E.; Tominaga, A.; Waki, H.; Miseki, K.; Tomioka, A.; Nakajima, K.; Kakehi, K.; Suzuki, M.; Taniguchi, N.; Suzuki, A. Structural Characterization of Monosialo-, Disialo- and Trisialo-Gangliosides by Negative Ion AP-MALDI-QIT-TOF Mass Spectrometry with MSn Switching. Neurochem. Res. 2012, 37 (6), 1315-1324. https://doi.org/10.1007/S11064012-0735-Z.

(19) Caprioli, R. M.; Farmer, T. B.; Gile, J. Molecular Imaging of Biological Samples: Localization of Peptides and Proteins Using MALDI-TOF MS. Anal. Chem. 1997, 69 (23), 4751-4760. https://doi.org/10.1021/AC970888I.

(20) El-Aneed, A.; Cohen, A.; Banoub, J. Mass Spectrometry, Review of the Basics: Electrospray, MALDI, and Commonly Used Mass Analyzers. Appl. Spectrosc. Rev. 2009, 44 (3), 210-230. https://doi.org/10.1080/05704920902717872.

(21) Mikula, S.; Elston, G.; Biswavas Misra, B.; Whitehead, S. N.; Weishaupt, N.; Caughlin, S.; K-C Yeung, K. Differential Anatomical Expression of Ganglioside GM1 Species Containing D18:1 or D20:1 Sphingosine Detected by MALDI Imaging Mass Spectrometry in Mature Rat Brain. 2015. https://doi.org/10.3389/fnana.2015.00155.

(22) Caughlin, S.; Park, D. H.; Yeung, K. K.-C.; Cechetto, D. F.; Whitehead, S. N. Sublimation of DAN Matrix for the Detection and Visualization of Gangliosides in Rat Brain Tissue for MALDI Imaging Mass Spectrometry. J. Vis. Exp. JOVE 2017, 2017 (121), 55254. https://doi.org/10.3791/55254.

(23) Yang, E.; Dufresne, M.; Chaurand, P. Enhancing Ganglioside Species Detection for MALDI-TOF Imaging Mass Spectrometry in Negative Reflectron Mode. Int. J. Mass Spectrom. 2019, 437, 3-9. https://doi.org/10.1016/j.ijms.2017.09.011.

(24) Colsch, B.; Jackson, S. N.; Dutta, S.; Woods, A. S. Molecular Microscopy of Brain Gangliosides: Illustrating Their Distribution in Hippocampal Cell Layers. ACS Chem. Neurosci. 2011, 2 (4), 213-222. https://doi.org/10.1021/cn100096h.

(25) Jackson, S. N.; Ugarov, M.; Egan, T.; Post, J. D.; Langlais, D.; Schultz, J. A.; Woods, A. S. MALDI-Ion Mohility-TOFMS Imaging of Lipids in Rat Brain Tissue. J. Mass Spectrom. 2007, 42 (8), 1093-1098. https://doi.org/10.1002/jms.1245.

(26) N. Jackson, S.; Benoit Colsch; Thomas Egan; K. Lewis, E.; Albert Schultz, J.; S. Woods, A. Gangliosides' Analysis by MALDI-Ion Mobility MS. Analyst 2011, 136 (3), 463-466. https://doi.org/10.1039/C0AN00732C.

(27) Tobias, F.; Pathmasiri, K. C.; Cologna, S. M. Mass Spectrometry Imaging Reveals Ganglioside and Ceramide Localization Patterns during Cerebellar Degeneration in the Npc1-/Mouse Model. Anal. Bioanal. Chem. 2019, 411 (22), 56595668. https://doi.org/10.1007/S00216-019-01989-7.

(28) Hájek, R.; Jirásko, R.; Lísa, M.; Cífková, E.; Holčapek, M. Hydrophilic Interaction Liquid Chromatography-Mass Spectrometry Characterization of Gangliosides in Biological Samples. Anal. Chem. 2017, 89 (22), 12425-12432. https://doi.org/10.1021/acs.analchem.7b03523.

(29) May, J. C.; Goodwin, C. R.; Lareau, N. M.; Leaptrot, K. L.; Morris, C. B.; Kurulugama, R. T.; Mordehai, A.; Klein, C.; Barry, W.; Darland, E.; Overney, G.; Imatani, K.; Stafford, G. C.; Fjeldsted, J. C.; McLean, J. A. Conformational Ordering of Biomolecules in the Gas Phase: Nitrogen Collision Cross Sections Measured on a Prototype High Resolution Drift Tube Ion Mobility-Mass Spectrometer. Anal. Chem. 2014, 86 (4), 2107-2116. https://doi.org/10.1021/ac4038448.

(30) Valentine, S. J.; Koeniger, S. L.; Clemmer, D. E. A Split-Field 
Drift Tube for Separation and Efficient Fragmentation of Biomolecular Ions. Anal. Chem. 2003, 75 (22), 6202-6208. https://doi.org/10.1021/ac030111r.

(31) Zhong, Y.; Hyung, S.-J.; Ruotolo, B. T. Characterizing the Resolution and Accuracy of a Second-Generation TravelingWave Ion Mobility Separator for Biomolecular Ions. Analyst 2011, 136 (17), 3534. https://doi.org/10.1039/c0an00987c.

(32) Shvartsburg, A. A.; Smith, R. D. Fundamentals of Traveling Wave Ion Mobility Spectrometry. Anal. Chem. 2008, 80 (24), 9689-9699. https://doi.org/10.1021/ac8016295.

(33) Deng, L.; Ibrahim, Y. M.; Baker, E. S.; Aly, N. A.; Hamid, A. M.; Zhang, X.; Zheng, X.; Garimella, S. V. B.; Webb, I. K.; Prost, S. A.; Sandoval, J. A.; Norheim, R. V.; Anderson, G. A.; Tolmachev, A. V.; Smith, R. D. Ion Mobility Separations of Isomers Based upon Long Path Length Structures for Lossless Ion Manipulations Combined with Mass Spectrometry. ChemistrySelect 2016, 1 (10), 2396-2399. https://doi.org/10.1002/slct.201600460.

(34) Ibrahim, Y. M.; Hamid, A. M.; Deng, L.; Garimella, S. V. B.; Webb I. K.; Baker, E. S.; Smith, R. D. New Frontiers for Mass Spectrometry Based upon Structures for Lossless Ion Manipulations. Analyst. 2017, 142 (7), 1010-1021. https://doi.org/10.1039/c7an00031f.

(35) Purves, R. W.; Guevremont, R. Electrospray Ionization HighField Asymmetric Waveform Ion Mobility Spectrometry-Mass Spectrometry. Int. J. Mass Spectrom. Ion Process. 1970, 52 (3), 2346-2357. https://doi.org/10.1021/ac981380y.

(36) Kolakowski, B. M.; Mester, Z. Review of Applications of HighField Asymmetric Waveform Ion Mobility Spectrometry (FAIMS) and Differential Mobility Spectrometry (DMS). $\begin{array}{llll}\text { Analyst } & \mathbf{2 0 0 7}, & 132 & \text { (9), }\end{array}$ https://doi.org/10.1039/b706039d.

(37) Guevremont, R. High-Field Asymmetric Waveform Ion Mobility Spectrometry: A New Tool for Mass Spectrometry. J. Chromatogr. A. 2004, 1058 (1-2), 3-19. https://doi.org/10.1016/j.chroma.2004.08.119.

(38) Fernandez-Lima, F.; Kaplan, D. A.; Suetering, J.; Park, M. A Gas-Phase Separation Using a Trapped Ion Mobility Spectrometer. Int. J. Ion Mobil. Spectrom. 2011, 14 (2-3). https://doi.org/10.1007/s12127-011-0067-8.

(39) Michelmann, K.; Silveira, J. A.; Ridgeway, M. E.; Park, M. A Fundamentals of Trapped Ion Mobility Spectrometry. J. Am. Soc. Mass Spectrom. 2015, 26 (1), 14-24. https://doi.org/10.1007/s13361-014-0999-4.

(40) Rivera, E. S.; Djambazova, K. V.; Neumann, E. K.; Caprioli, R. M.; Spraggins, J. M. Integrating Ion Mobility and Imaging Mass Spectrometry for Comprehensive Analysis of Biological Tissues: A Brief Review and Perspective. J. Mass Spectrom. 2020, 55 (12), e4614. https://doi.org/10.1002/JMS.4614.

(41) Sans, M.; Feider, C. L.; Eberlin, L. S. Advances in Mass Spectrometry Imaging Coupled to Ion Mobility Spectrometry for Enhanced Imaging of Biological Tissues. Curr. Opin. Chem. Biol. 2018, 42, 138-146. https://doi.org/10.1016/j.cbpa.2017.12.005.

(42) Sarbu, M.; Robu, A. C.; Ghiulai, R. M.; Vukelić, Ž.; Clemmer, D. E.; Zamfir, A. D. Electrospray Ionization Ion Mobility Mass Spectrometry of Human Brain Gangliosides. Anal. Chem. 2016, 88 (10), 5166-5178. https://doi.org/10.1021/ACS.ANALCHEM.6B00155.

(43) Sarbu, M.; Clemmer, D. E.; Zamfir, A. D. Ion Mobility Mass Spectrometry of Human Melanoma Gangliosides. Biochimie 2020, 177, 226-237. https://doi.org/10.1016/J.BIOCHI.2020.08.011.

(44) Sarbu, M.; Petrica, L.; Clemmer, D. E.; Vukelić, Ž.; Zamfir, A. D. Gangliosides of Human Glioblastoma Multiforme: A Comprehensive Mapping and Structural Analysis by Ion Mobility Tandem Mass Spectrometry. J. Am. Soc. Mass Spectrom. 2021, 32 (5), 1249-1257. https://doi.org/10.1021/jasms.1c00088.

(45) Wormwood Moser, K. L.; Van Aken, G.; DeBord, D.; Hatcher, N. G.; Maxon, L.; Sherman, M.; Yao, L.; Ekroos, K. High-Defined
Quantitative Snapshots of the Ganglioside Lipidome Using High Resolution Ion Mobility SLIM Assisted Shotgun Lipidomics. Anal. Chim. Acta. 2021, 1146, 77-87. https://doi.org/10.1016/j.aca.2020.12.022.

(46) Jackson, S. N.; Muller, L.; Roux, A.; Oktem, B.; Moskovets, E.; Doroshenko, V. M.; Woods, A. S. AP-MALDI Mass Spectrometry Imaging of Gangliosides Using 2,6Dihydroxyacetophenone. J. Am. Soc. Mass Spectrom 2018, 29, 1463-1472. https://doi.org/10.1007/s13361-018-1928-8. Hernandez, D. R.; DeBord, J. D.; Ridgeway, M. E.; Kaplan, D. A.; Park, M. A.; Fernandez-Lima, F. Ion Dynamics in a Trapped Ion Mobility Spectrometer. Analyst. 2014, 139 (8), 1913-1921. https://doi.org/10.1039/C3AN02174B.

(48) Ridgeway, M. E.; Lubeck, M.; Jordens, J.; Mann, M.; Park, M. A. Trapped Ion Mobility Spectrometry: A Short Review. Int. J. Mass Spectrom. 2018, 425, 22-35. https://doi.org/10.1016/J.IJMS.2018.01.006.

(49) Silveira, J. A.; Ridgeway, M. E.; Park, M. A. High Resolution Trapped Ion Mobility Spectrometery of Peptides. Anal. Chem. 2014, $86 \quad$ (12), 5624-5627. https://doi.org/10.1021/ac501261h

(50) Spraggins, J. M.; Djambazova, K. V.; Rivera, E. S.; Migas, L. G.; Neumann, E. K.; Fuetterer, A.; Suetering, J.; Goedecke, N.; Ly, A.; Van De Plas, R.; Caprioli, R. M. High-Performance Molecular Imaging with MALDI Trapped Ion-Mobility Timeof-Flight (TimsTOF) Mass Spectrometry. Anal. Chem. 2019, 91 (22), $14552-14560$ https://doi.org/10.1021/acs.analchem.9b03612.

(51) Djambazova, K.; Klein, D. R.; Migas, L. G.; Neumann, E. K.; Rivera, E. S.; Van de Plas, R.; Caprioli, R. M.; Spraggins, J. M. Resolving the Complexity of Spatial Lipidomics Using MALDI TIMS Imaging Mass Spectrometry. Anal. Chem. 2020. https://doi.org/10.1021/acs.analchem.0c02520.

(52) Fu, T.; Oetjen, J.; Chapelle, M.; Verdu, A.; Szesny, M.; Chaumot A.; Degli-Esposti, D.; Geffard, O.; Clément, Y.; Salvador, A.; Ayciriex, S. In Situ Isobaric Lipid Mapping by MALDI-Ion Mobility Separation-Mass Spectrometry Imaging. J. Mass Spectrom. 2020, e4531. https://doi.org/10.1002/jms.4531.

(53) Palmer-Toy, D. E.; Sarracino, D. A.; Sgroi, D.; Levangie, R.; Leopold, P. E.; Xu, B. J.; Caprioli, R. M.; Sanders, M. E.; Jensen, R. A.; Bhattacharya, S. H.; Gal, A. A.; Murray, K. K. Integrating Histology and Imaging Mass Spectrometry. J. Am. Soc. Mass $\begin{array}{llll}\text { Spectrom } & \mathbf{2 0 0 3} & 3 & (10),\end{array}$ https://doi.org/10.1021/ac0351264.

(54) Lein, E. S.; Hawrylycz, M. J.; Ao, N.; Ayres, M.; Bensinger, A.; Bernard, A.; Boe, A. F.; Boguski, M. S.; Brockway, K. S.; Byrnes, E. J.; Chen, L.; Chen, L.; Chen, T.-M.; Chi Chin, M.; Chong, J.; Crook, B. E.; Czaplinska, A.; Dang, C. N.; Datta, S.; Dee, N. R.; Desaki, A. L.; Desta, T.; Diep, E.; Dolbeare, T. A.; Donelan, M. J.; Dong, H.-W.; Dougherty, J. G.; Duncan, B. J.; Ebbert, A. J.; Eichele, G.; Estin, L. K.; Faber, C.; Facer, B. A.; Fields, R.; Fischer, S. R.; Fliss, T. P.; Frensley, C.; Gates, S. N.; Glattfelder, K. J.; Halverson, K. R.; Hart, M. R.; Hohmann, J. G.; Howell, M. P.; Jeung, D. P.; Johnson, R. A.; Karr, P. T.; Kawal, R.; Kidney, J. M.; Knapik, R. H.; Kuan, C. L.; Lake, J. H.; Laramee, A. R.; Larsen, K. D.; Lau, C.; Lemon, T. A.; Liang, A. J.; Liu, Y.; Luong, L. T.; Michaels, J.; Morgan, J. J.; Morgan, R. J.; Mortrud, M. T. Mosqueda, N. F.; Ng, L. L.; Ng, R.; Orta, G. J.; Overly, C. C.; Pak, T. H.; Parry, S. E.; Pathak, S. D.; Pearson, O. C.; Puchalski, R. B.; Riley, Z. L.; Rockett, H. R.; Rowland, S. A.; Royall, J. J.; Ruiz, M. J.; Sarno, N. R.; Schaffnit, K.; Shapovalova, N. V.; Sivisay, T.; Slaughterbeck, C. R.; Smith, S. C.; Smith, K. A.; Smith, B. I.; Sodt A. J.; Stewart, N. N.; Stumpf, K.-R.; Sunkin, S. M.; Sutram, M.; Tam, A.; Teemer, C. D.; Thaller, C.; Thompson, C. L.; Varnam, L. R.; Visel, A.; Whitlock, R. M.; Wohnoutka, P. E.; Wolkey, C. K.; Wong, V. Y.; Wood, M.; Yaylaoglu, M. B.; Young, R. C.; Youngstrom, B. L.; Feng Yuan, X.; Zhang, B.; Zwingman, T. A.; Jones, A. R. Genome-Wide Atlas of Gene Expression in the Adult Mouse Brain. Nature 2006, 445 (7124), 168-176. https://doi.org/10.1038/nature05453.

(55) Ng, L.; Bernard, A.; Lau, C.; Overly, C. C.; Dong, H.-W.; Kuan, C.; 
Pathak, S.; Sunkin, S. M.; Dang, C.; Bohland, J. W.; Bokil, H.; Mitra, P. P.; Puelles, L.; Hohmann, J.; Anderson, D. J.; Lein, E. S.; Jones, A. R.; Hawrylycz, M. An Anatomic Gene Expression Atlas of the Adult Mouse Brain. Nat. Neurosci. 2009, 12 (3), 356-362. https://doi.org/10.1038/nn.2281.

(56) Spraggins, J. M.; Rizzo, D. G.; Moore, J. L.; Rose, K. L.; Hammer, N. D.; Skaar, E. P.; Caprioli, R. M. MALDI FTICR IMS of Intact Proteins: Using Mass Accuracy to Link Protein Images with Proteomics Data. J. Am. Soc. Mass Spectrom 2015, 26 (6), 947985. https://doi.org/10.1007/s13361-015-1147-5.

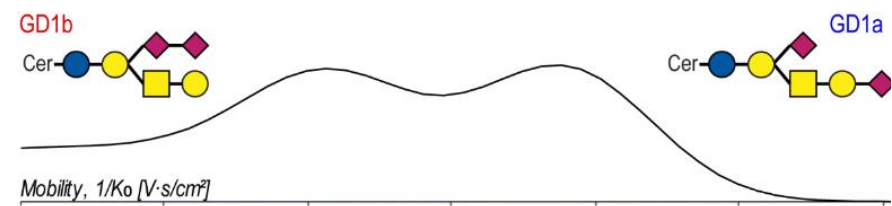

(57)

Watson, C.; Paxinos, G.; Kayalioglu, G.; Heise, C. Chapter 15 Atlas of the Rat Spinal Cord. In The Spinal Cord; Academic Press, 2008; pp 238-306. https://doi.org/10.1016/B978-012-374247-6.50019-5.

(58) Kotani, M.; Kawashima, D.; Ozawa, H.; Terashima, T.; Tai, T. Differential Distribution of Major Gangliosides in Rat Central Nervous System Detected by Specific Monoclonal Antibodies; 1993; Vol. 3.

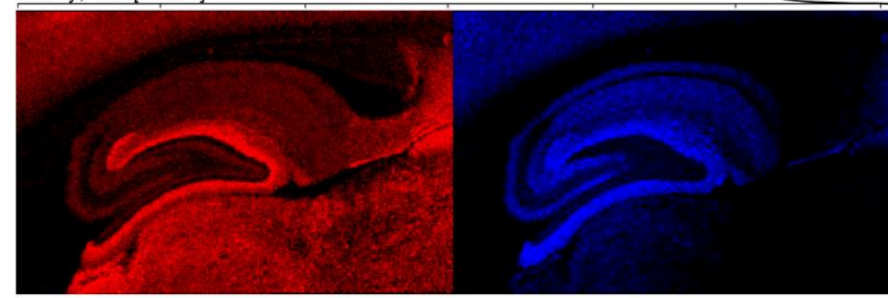

